\title{
Systemic therapy for advanced cholangiocarcinoma: new options on the horizon
}

\author{
Saleh A. Alqahtani ${ }^{1,2}$, Massimo Colombo ${ }^{3}$ \\ 'Liver Transplant Center, King Faisal Specialist Hospital \& Research Center, Riyadh 12713, Saudi Arabia. \\ 2Division of Gastroenterology and Hepatology, Johns Hopkins University, Baltimore, MD 21287, USA. \\ ${ }^{3}$ Liver Center, San Raffaele Hospital, Milan 20132, Italy.
}

Correspondence to: Dr. Saleh A. Alqahtani, Division of Gastroenterology and Hepatology, Johns Hopkins University, 600 North Wolfe St, Blalock Suite 412, Baltimore, MD 21287, USA. E-mail: salqaht1@jhmi.edu

How to cite this article: Alqahtani SA, Colombo M. Systemic therapy for advanced cholangiocarcinoma: new options on the horizon. Hepatoma Res 2020;6:70. http://dx.doi.org/10.20517/2394-5079.2020.65

Received: 28 Jun 2020 First Decision: 2 Sep 2020 Revised: 3 Sep 2020 Accepted: 9 Sep 2020 Published: 12 Oct 2020

Academic Editor: Shu-Kui Qin Copy Editor: Cai-Hong Wang Production Editor: Jing Yu

\begin{abstract}
Patients with unresectable cholangiocarcinoma (CCA) face a poor prognosis, and there are few effective treatment options for the disease. The standard of care for patients with locally advanced or metastatic CCA is chemotherapy with a gemcitabine-based doublet. Unfortunately, the clinical benefit obtained with these regimens is modest, with a median overall survival of about one year. For CCA that is chemotherapy-refractory or recurs after first-line chemotherapy, the treatment options are even more limited, and no relevant randomized controlled data are available. In recent years, molecular profiling has shed light on the molecular basis of CCA and identified subgroups of patients that might benefit from a personalized treatment approach. These efforts resulted in the recent FDA approval of the fibroblast growth factor receptor (FGFR) inhibitor, pemigatinib, as a second-line treatment for patients with advanced CCA harboring an FGFR2-fusion or rearrangement. Several other targeted agents also are under evaluation in patients with CCA, of which the isocitrate dehydrogenase inhibitor has had the most promising results. Finally, immunotherapy is being explored as a new treatment approach for advanced CCA patients; indeed, the immune checkpoint inhibitor pembrolizumab can already be used to treat CCAs that are mismatch repair deficient. This review is a comprehensive overview of the treatment options for CCA and offers a glimpse into what the future could hold for these patients.
\end{abstract}

Keywords: Cholangiocarcinoma, fibroblast growth factor receptor inhibitor, isocitrate dehydrogenase inhibitor, immune checkpoint inhibitor 


\section{INTRODUCTION}

Cholangiocarcinoma (CCA) refers to a group of malignancies that arise from epithelial cells along the biliary tree ${ }^{[1]}$. It is a rare tumor type that accounts for less than $1 \%$ of all human cancers ${ }^{[1]}$. Based on the location of the tumor, CCAs are divided into three categories: Intrahepatic CCAs (iCCAs) are within the liver parenchyma (i.e., proximal to the second-degree bile ducts), whereas perihilar CCAs (pCCAs) and distal CAAs (dCCAs) are outside the liver, with the cystic duct as the boundary between the two types ${ }^{[1]}$. Most CCAs are extrahepatic; iCCAs account for only $10 \%-20 \%$ of cases ${ }^{[2,3]}$. CCAs are classified histopathologically as adenocarcinomas, but rare histologic subtypes can be encountered ${ }^{[4]}$.

Over the last decades, the incidence of CCA has increased in Western countries ${ }^{[3]}$, which is the basis for large studies that have looked into risk factors for cancer development ${ }^{[5,6]}$. The most prominent risk factors identified in these analyses are liver cirrhosis, viral hepatitis, metabolic syndrome, and diabetes mellitus ${ }^{[5-8]}$. However, the best-known risk factors for CCA are pre-existing conditions, such as choledochal cysts, inflammatory bowel disease, and primary sclerosing cholangitis ${ }^{[9,10]}$. The incidence of CCA is highest in Southeast Asia, where there is a strong relationship between infections with the hepatobiliary flukes Opisthorchis viverrini and Clonorchis sinensis and $\mathrm{CCA}^{[10]}$.

The only potentially curative treatment for patients with CCA is radical surgical resection of the lesion combined with lymphadenectomy ${ }^{[3]}$. Unfortunately, however, surgical resection is feasible in only about $30 \%$ of patients ${ }^{[3,11]}$, and recurrence after surgery is frequent; thus the prospects of long-term survival after resection are poor ${ }^{[2,3,11]}$. To counter the high rates of local and distant recurrence after surgery for CCA, several adjuvant treatment strategies have been explored, with mixed results ${ }^{[3,12-16]}$. In about $70 \%$ of patients, the disease is unresectable or metastatic at the time of diagnosis ${ }^{[1]}$. For these patients, the treatment options are usually limited to systemic therapies ${ }^{[1,3]}$. Only in a minority of advanced CCA patients are palliative loco-regional therapies beneficial, and the use of this approach is restricted mainly to patients with iCCA whose disease spread is limited to the liver. In this setting, small studies have demonstrated that transarterial chemo- or radioembolization can provide local disease control, with a survival benefit comparable to that of supportive care ${ }^{[17-21]}$. More recently, insight into the molecular basis of CCA and understanding of the interplay between tumor cells and the immune system have led to the development of targeted treatments. The most promising results in this area have come from studies evaluating inhibitors of mutated forms of the FGFR or isocitrate dehydrogenase $(I D H)$ and from studies evaluating immune checkpoint inhibitors.

This review provides an overview of the systemic treatment options for patients with advanced CCA. Data from the use of chemotherapy regimens in initial treatment and of recurrent disease, as well as a summary of the clinical trials evaluating molecularly targeted agents or immunotherapy, are presented.

\section{IS THERE A PLACE FOR ADJUVANT CHEMOTHERAPY IN PATIENTS WITH SURGICALLY RESECTED CCA?}

As discussed above, surgical resection of CCA is associated with a high rate of disease recurrence and a poor long-term survival rate. In a series of 564 CCA patients who were operated on between 1973 and 2004, the five-year overall survival (OS) rate was only $18 \%$ (30\% in patients in whom an Ro resection was possible; median OS 15 months for all patients, 28 months for Ro patients ${ }^{[2]}$. The survival rate was better in patients with more proximal tumors (i.e., five-year OS rates for Ro patients with iCCA, pCCA, and dCCA of $63 \%, 30 \%$, and $27 \%$, respectively $)^{[2]}$. Data from the Memorial Sloan-Kettering Cancer Center confirm the high risk of disease recurrence after surgical resection of CCAs; in their series, the median disease-specific survival of patients with resected CCA was only 36 months, and almost two-thirds of patients had disease relapse during a median follow-up of 26 months $^{[11]}$. In attempts to improve the dismal prognosis of resected 
CCA patients, adjuvant treatment strategies have been explored, including chemotherapy, radiotherapy, and combination chemoradiotherapy.

A systematic review and meta-analysis of data of 6,712 patients with CCA of the gallbladder or biliary ducts who received adjuvant therapy with either chemotherapy, radiotherapy, or chemoradiotherapy after surgery revealed a non-significant OS improvement compared to OS with surgery alone $(P=0.06)^{[16]}$. Patients who received chemo- or chemoradiotherapy had a significantly greater survival benefit than patients who received adjuvant radiotherapy alone $(\mathrm{OR}=0.39,0.61 \text {, and } 0.98 \text {, respectively, } P=0.02)^{[16]}$. A second metaanalysis, reported by Ghidini et al. ${ }^{[22]}$ also found a survival benefit from adjuvant chemo(radio)therapy in patients with resected biliary tract cancers; in that analysis $(n=22,499)$, adjuvant therapy was associated with a significant (4.3 months) prolongation in median OS. Compared with surgery alone, adjuvant chemo (radio)therapy was associated with a $41 \%$ reduced risk of death $(\mathrm{HR}=0.59,95 \% \mathrm{CI}$ : $0.49-0.71, P<0.001)$

${ }^{[22]}$. More recently, however, two prospective, randomized phase III trials of CCA patients found no clinical benefit from adjuvant chemotherapy. The PRODIGE-12 trial randomized 196 patients with localized biliary tract cancer to observation or adjuvant chemotherapy with the GEMOX regimen (gemcitabine $1000 \mathrm{mg} /$ $\mathrm{m}^{2}$ on Day 1 and oxaliplatin $85 \mathrm{mg} / \mathrm{m}^{2}$ infused on Day 2 of a two-week cycle for 12 cycles). Patients who received adjuvant GEMOX had a median recurrence-free survival of 30.4 months compared with 18.5 months for patients randomized to the control arm. However, this numerical difference did not meet the threshold for statistical significance $(\mathrm{HR}=0.88,95 \% \mathrm{CI}: 0.62-1.25, P=0.48)$. In addition, there was no significant difference in OS (median OS: 75.8 months vs. 50.8 months; $\mathrm{HR}=1.08,95 \% \mathrm{CI}$ : 0.70-1.66, $P=0.74$ ) ${ }^{[12]}$. Similarly, a randomized phase III trial comparing adjuvant gemcitabine alone to observation in 225 patients with resected bile duct cancer found no difference in OS (median OS: 62.3 months vs. 63.8 months; $\mathrm{HR}=1.01,95 \% \mathrm{CI}$ : $0.70-1.15, P=0.96$ ) or recurrence-free survival (median: 36.0 months vs. 39.9 months; $\mathrm{HR}=0.93,95 \% \mathrm{CI}: 0.66-1.32, P=0.69)^{[13]}$.

In the phase III BILCAP trial, 447 patients with histologically confirmed CCA or muscle-invasive gallbladder cancer who underwent a complete resection were randomized to receive oral capecitabine (1250 mg/m² BID on Days 1-14 of a 21-day cycle, for eight cycles) or observation. After a median followup of 60 months, the median OS for patients in the adjuvant chemotherapy arm was 51.1 months, which was almost 15 months longer than the 36.4 median OS in the observation arm. In a protocol-specified sensitivity analysis, this difference in OS was statistically significant, with an HR of 0.71 (95\%CI: 0.55-0.92, $P=0.010)^{[14]}$. Although the trial failed to meet the primary endpoint of improving OS in the intentionto-treat population, the prespecified sensitivity and per-protocol analyses showed signals of capecitabine efficacy and could be considered for adjuvant care. Based on these results, capecitabine has become the preferred adjuvant chemotherapy regimen in patients with resected CCA. In addition, the American Society of Clinical Oncology endorsed this adjuvant chemotherapy regimen in its 2019 practice guidelines update ${ }^{[23]}$. In line with endorsement, the ongoing ACTICCA-1 trial, evaluating adjuvant gemcitabinecisplatin in patients with resected CCA or muscle-invasive gallbladder cancer, amended its protocol and changed its control arm from observation to capecitabine ${ }^{[15]}$. The results of this trial are awaited.

\section{CHEMOTHERAPY FOR UNRESECTABLE OR METASTATIC CCA}

\section{First-line therapy}

As long ago as 1996, it was established that chemotherapy could improve the survival rate and quality of life of patients with advanced biliary tract cancer $^{[24]}$. In the early 2000s, gemcitabine monotherapy was often used as a frontline regimen for patients with advanced CCA. In a phase II trial $(n=23)$, gemcitabine $\left(1000 \mathrm{mg} / \mathrm{m}^{2}\right.$ over $60 \mathrm{~min}$ once a week in a two-weeks on/one-week off schedule) resulted in a median progression-free survival (PFS) of 8.1 months with a median OS of 13.1 months $^{[25]}$. However, in a retrospective case series of 100 patients with advanced CCA, the results obtained with gemcitabine monotherapy did not match the results of this phase II trial, with a median OS of only 7.3 months, and only one, out of five patients, was alive after one year ${ }^{[26]}$. 
Table 1. Chemotherapy in the first-line treatment of patients with advanced CCA: an overview of pivotal clinical trials

\begin{tabular}{|c|c|c|c|c|c|}
\hline Trial & Phase & Regimen & $\mathbf{N}$ & $\begin{array}{c}\text { Median OS } \\
{[\mathrm{HR}(95 \% \mathrm{Cl})]}\end{array}$ & Response rate \\
\hline$A B C-02^{[29]}$ & III & Gem-Cis vs. Gem & 410 & $\begin{array}{l}11.7 \text { months vs. } 8.1 \text { months } \\
0.640(52-0.80)\end{array}$ & DCR: $81.4 \%$ vs. $71.8 \%$ \\
\hline Okusaka et al. ${ }^{[30]}$ & II & Gem-Cis vs. Gem & 83 & $\begin{array}{l}11.2 \text { months vs. } 7.7 \text { months } \\
0.69(0.42-1.13)\end{array}$ & $\begin{array}{l}\text { ORR: } 19.5 \% \text { vs. } 11.9 \% \\
\text { DCR: } 68.3 \% \text { vs. } 50.0 \%\end{array}$ \\
\hline FUGA-BT ${ }^{[32]}$ & III & Gem-Cis vs. Gem-S1 & 354 & $\begin{array}{l}13.4 \text { months vs. } 15.1 \text { months } \\
0.945(0.78-1.15)^{\star}\end{array}$ & ORR: $29.8 \%$ vs. $32.4 \%$ \\
\hline $\operatorname{GERCOR}^{[33] \#}$ & II & GEMOX & 33 & 15.4 months & ORR: $36 \%$ \\
\hline Kim et al. ${ }^{[34]}$ & III & GEMOX vs. XELOX & 222 & $\begin{array}{l}10.4 \text { months vs. } 10.6 \text { months } \\
\text { NR }\end{array}$ & $\begin{array}{l}\text { ORR: } 24.6 \% \text { vs. } 15.7 \% \\
\text { DCR: } 63.2 \% \text { vs. } 58.3 \%\end{array}$ \\
\hline Shroff et al. ${ }^{[36]}$ & II & Gem-Cis + nab-paclitaxel & 60 & 19.2 months & $\begin{array}{l}\text { ORR: } 45 \% \\
\text { DCR: } 84 \%\end{array}$ \\
\hline KHBO1401-MITSUBA ${ }^{[37]}$ & III & Gem-Cis vs. Gem-Cis-S1 & 246 & $\begin{array}{l}13.5 \text { months vs. } 12.6 \text { months } \\
0.79(0.60-1.04)\end{array}$ & ORR: $41.5 \%$ vs. $15 \%$ \\
\hline
\end{tabular}

*90\% confidence interval. Study met threshold for non-inferiority; ${ }^{*}$ only data for Group A (good performance status) are listed here. CCA: cholangiocarcinoma; DCR: disease control rate; ORR: overall response rate; OS: overall survival

Table 1 lists the pivotal clinical trials which study various chemotherapy options in the first-line treatment of patients with advanced CCA.

\section{Gemcitabine-based doublet chemotherapy}

To improve on these outcomes, numerous gemcitabine-based combination regimens have been tested. The most prominent consists of the gemcitabine-cisplatin (Gem-Cis) doublet. Two phase-II trials of the combination produced efficacy signals in patients with advanced CCA and had a favorable toxicity profile ${ }^{[27,28]}$. These encouraging findings formed the rationale for comparing the Gem-Cis doublet to gemcitabine alone in a randomized phase III trial: In the ABC-02 study, 410 patients with locally advanced or metastatic CCA, gallbladder cancer, or ampullary cancer were randomly assigned to receive cisplatin $\left(25 \mathrm{mg} / \mathrm{m}^{2}\right)$ followed by gemcitabine $\left(1000 \mathrm{mg} / \mathrm{m}^{2}\right.$ on Days 1 and 8 , every three weeks for eight cycles) or gemcitabine alone (1000 $\mathrm{mg} / \mathrm{m}^{2}$ on Days 1,8 , and 15 , every four weeks for six cycles) for a total of 24 weeks. Study patients who received the combination treatment had a median OS of 11.7 months, which was significantly longer than 8.1 months in the gemcitabine-treated cohort $(\mathrm{HR}=0.64,95 \% \mathrm{CI}: 0.52-0.80, P<$ 0.001 ); the PFS also was significantly longer (median PFS: 8 months $v s .5$ months; $P<0.001$ ) in patients treated with the chemotherapy doublet, and the tumor was controlled in significantly more patients (81.4\% vs. $71.8 \% ; P=0.049)^{[29]}$. Toxicity was similar with the two treatments, but the addition of cisplatin to the regimen resulted in more Grade 3/4 neutropenia (25\% vs. 17\%) and a higher incidence of Grade 3/4 liver abnormalities $(27 \% \text { vs. } 17 \%)^{[29]}$. A similar efficacy benefit of Gem-Cis over gemcitabine alone was reported by Okusaka et al. ${ }^{[30]}$, with a median OS of 11.2 months with the combination compared with 7.7 months with gemcitabine alone $(\mathrm{HR}=0.69,95 \% \mathrm{CI}$ : 0.42-1.13); at the one-year mark, this difference translated into an absolute survival difference of $8 \%{ }^{[30]}$. A subsequent meta-analysis of these two studies indicated that, compared to gemcitabine alone, Gem-Cis was associated with a $35 \%$ reduced death risk $(\mathrm{HR}=0.65,95 \% \mathrm{CI}$ : $0.54-0.78, P<0.001)$ and a $36 \%$ reduced risk for disease progression $(\mathrm{HR}=0.64,95 \% \mathrm{CI}$ : 0.53-0.76, $P<$ 0.001). The benefit of Gem-Cis over gemcitabine monotherapy was present irrespective of the location of the primary tumor (i.e., gallbladder or CCA). These findings established Gem-Cis as the reference first-line treatment for patients with advanced CCA. However, a subgroup analysis of the performance status found that the superiority of Gem-Cis over gemcitabine alone was mainly in patients with good performance status, whereas patients with a European Cooperative Oncology Group (ECOG) performance status of 2 or more benefited less ${ }^{[31]}$.

A second gemcitabine-based doublet regimen that has gained momentum in recent years is the combination of gemcitabine and S-1, an oral fluoropyrimidine that includes three agents (tegafur, gimeracil, 
and oteracil) (Gem-S). In the Japanese FUGA-BT trial, 354 chemotherapy-naïve patients with recurrent or unresectable biliary tract cancer and an ECOG performance status of 0-1 were randomized to treatment with gemcitabine $\left(1000 \mathrm{mg} / \mathrm{m}^{2}\right.$ on Days 1 and 8 ) in combination with either S-1 (60, 80, or $100 \mathrm{mg}$ per day on Days 1-14 of a 21-day cycle) or cisplatin (25 mg/m IV on Days 1 and 8). In this study, Gem-S was noninferior to Gem-Cis, with a median OS of 15.1 and 13.4 months, respectively (HR $=0.945,90 \% \mathrm{CI}$ : 0.78-1.15, non-inferiority $P=0.046)^{[32]}$. In addition, Gem-Cis and Gem-S yielded similar results in PFS (median PFS: 5.8 months vs. 6.8 months; $\mathrm{HR}=0.86,95 \% \mathrm{CI}$ : 0.70-1.07) and overall response rate (ORR): $32.4 \%$ vs. $29.8 \%$. Clinically significant adverse events (AEs) were reported by $35.1 \%$ of patients enrolled in the Gem-Cis compared with $29.9 \%$ in the Gem-S arm. Based on these findings, Gem-S, when available, is a feasible firstline alternative to Gem-Cis in patients with advanced CCA.

Other gemcitabine-based doublets have also been evaluated in patients with advanced CCA. In the phase II GERCOR trial, 33 patients with newly diagnosed advanced biliary tract cancer and good performance status were treated with a combination of gemcitabine $\left(1000 \mathrm{mg} / \mathrm{m}^{2}\right.$ as a $10 \mathrm{mg} / \mathrm{m}^{2} / \mathrm{min}$ infusion on Day 1) and oxaliplatin $\left(100 \mathrm{mg} / \mathrm{m}^{2}\right.$ as a 2-h infusion on Day 2), every two weeks (GEMOX). The GEMOX regimen induced an ORR of $36 \%$ and had a median PFS and OS of 5.7 months and 15.4 months, respectively ${ }^{[33]}$. Recently, the GEMOX regimen was evaluated in a phase III setting, where it was compared to a combination of capecitabine (1000 mg/m $\mathrm{m}^{2}$ BID on Days 1-14) and oxaliplatin (130 mg/m $\mathrm{m}^{2}$ on Day 1) (XELOX). In that non-inferiority trial, with 222 patients with advanced biliary cancer, GEMOX and XELOX were given every three weeks for eight cycles ${ }^{[34]}$. The median PFS for GEMOX and XELOX was 5.3 months and 5.8 months, respectively, translating to a five-month PFS rate of $44.5 \%$ with GEMOX and $46.7 \%$ with XELOX. OS was not significantly different in the two arms, with a median OS of 10.4 and 10.6 months for GEMOX and XELOX, respectively ${ }^{[34]}$. These two studies established the clinical efficacy of GEMOX in the frontline treatment of patients with advanced biliary tract cancer, and the drug has become widely used in the treatment of patients with advanced CCA. This practice was fueled by oxaliplatin having a more favorable toxicity profile than that of cisplatin. However, whether GEMOX is non-inferior or superior to Gem-Cis has not been established (no head-to-head comparisons).

A final gemcitabine-based doublet that was explored in patients with advanced CCA is gemcitabine plus nab-paclitaxel. In 2018, Sahai et al. ${ }^{[35]}$ reported the results of a multicenter phase II trial in which 74 patients with advanced CCA were treated with nab-paclitaxel $\left(125 \mathrm{mg} / \mathrm{m}^{2} \mathrm{IV}\right)$ followed by gemcitabine $\left(1000 \mathrm{mg} / \mathrm{m}^{2}\right.$ on Days 1, 8, and 15) in 28-day treatment cycles ${ }^{[35]}$. The regimen was effective, with an ORR of $30 \%$ and a median OS of 12.4 months. The most common high-grade AEs with the gemcitabine nab-paclitaxel combination were neutropenia ( $43 \%$ Grade $\geq 3$ ) and fatigue ( $14 \%$ Grade $\geq 3$ ). These findings are promising, but validation in a randomized comparison with the current standard of care (Gem-Cis or Gem-S) is required before this regimen can be used routinely.

\section{Treatment intensification: the more, the merrier?}

Several clinical trials have evaluated whether a more intensive treatment strategy would result in better treatment outcomes than those of the current two-drug standard in the frontline treatment of advanced biliary tract cancer. A phase II trial, conducted at the MD Anderson Cancer Center and the Mayo Clinic, tested a triple regimen of gemcitabine, cisplatin, and nab-paclitaxel (initially 1000, 25, and $125 \mathrm{mg} / \mathrm{m}^{2}$, respectively, on Days 1 and 8 of 21-day cycles, with a later reduction to 800,25 , and $100 \mathrm{mg} / \mathrm{m}^{2}$, respectively, to mitigate the hematological toxicity) in 60 patients with advanced biliary tract cancer $(78 \% \mathrm{CCA})^{[36]}$. The results with the triplet regimen were promising, with a median PFS of 11.8 months and a median OS of 19.2 months. Forty-five percent of patients obtained a partial response, and $39 \%$ had disease stabilization. As could be expected, this benefit came with the cost of substantial toxicity, with $58 \%$ of patients experiencing Grade $\geq 3$ AEs (Grade $\geq 3$ AE neutropenia was most common, present in $33 \%$ of patients). Sixteen percent of patients withdrew from the treatment because of toxicity ${ }^{[36]}$. This regimen will be evaluated further in a phase III randomized trial (NCT03768414). 
A second interesting treatment-intensification study in patients with biliary tract cancer is the phase III KHBO1401-MITSUBA trial. In that, 246 chemotherapy-naïve patients with advanced biliary tract adenocarcinoma were randomly assigned to treatment with Gem-Cis or a triple combination of Gem-Cis and S-1 (GCS). The addition of S- 1 to Gem-Cis resulted in a lengthening of the median OS from 12.6 months to 13.5 months $(\mathrm{HR}=0.79,95 \% \mathrm{CI}: 0.60-1.04, P=0.046)$. At the one-year mark, this translated into an absolute survival difference of $5.7 \%$ in favor of GCS (59.4\% vs. 53.7\%). In addition, the median PFS was significantly longer with GCS than with Gem-Cis (7.4 months vs. 5.5 months; HR = 0.75, 95\%CI: 0.58-0.97, $P=0.0015)$, and the rate of patients who had a treatment response was almost tripled $(41.5 \% v s .15 .0 \%)^{[37]}$. Based on these results, the authors concluded that GCS could become a new standard treatment for patients with advanced biliary tract cancer.

\section{Patients with poor performance status: can we do good enough with a little bit less?}

As indicated above, poor performance status seemed to be associated with a lower likelihood of treatment benefit from a Gem-Cis doublet. For these patients, gemcitabine monotherapy can be considered. 5-fluorouracil (5-FU) monotherapy is not recommended in patients with biliary tract cancer because of the low response rate (ORR: $20 \%)^{[38]}$, but response rates were slightly higher when leucovorin was used in combination with 5-FU: in 28 patients with biliary tract cancer, leucovorin-modulated 5-FU resulted in an ORR of $32.1 \%$, with a median OS of six months ${ }^{[39]}$. Similar results were reported by Chen et al. ${ }^{[40]}$ in a series of 19 biliary tract cancer patients (ORR: 33\%, median OS 7.0 months).

A second alternative for gemcitabine monotherapy in CCA patients with a poor performance status could be capecitabine monotherapy. In a study from the MD Anderson Cancer Center ${ }^{[41]}$, 63 patients with advanced hepatocellular carcinoma $(n=37)$, gallbladder cancer $(n=8)$, or CCA $(n=18)$ were treated with capecitabine monotherapy ( $1000 \mathrm{mg} / \mathrm{m}^{2}$ BID for 14 days, in 21 -day cycles). Among the CCA patients in this trial, a median OS of 8.1 months was reported. Although the response rate in this trial was modest, CCA patients have been reported to survive long term with capecitabine monotherapy ${ }^{[41]}$.

\section{Second-line therapy}

Few studies have been conducted in patients with advanced CCA and progression after first-line therapy, so there is no established standard of care for these persons. There are also few data on selecting patients who might benefit from second-line therapy; the available studies consistently required good performance status to initiate second-line therapy ${ }^{[42-45]}$. Other prognostic factors are the treatment effect in first-line therapy (disease control or not), a low CA19-9 level, and the absence of peritoneal carcinomatosis ${ }^{[44,45]}$.

In 2014, Lamarca et al ${ }^{[46]}$ published a systematic review of clinical studies that evaluated second-line chemotherapy (fluoropyrimidine, irinotecan, docetaxel, and gemcitabine) in patients with advanced biliary tract cancer. A platinum compound was often used in second-line therapy in patients who received a fluoropyrimidine in first-line treatment; the median OS in this analysis was 7.2 months, with a median PFS of 3.2 months. The response rate to second-line chemotherapy was only $7.7 \%{ }^{[46]}$. Currently, the most frequently used second-line treatment for patients with advanced CCA, who have failed first-line Gem-Cis, is the FOLFOX regimen (oxaliplatin plus 5-FU). This practice is based on the results of the randomized, phase III ABC-06 trial. One hundred and sixty-two patients with locally advanced or metastatic biliary tract cancer, who were previously treated with Gem-Cis, were randomly assigned to active symptom control with or without modified FOLFOX (mFOLFOX) regimen, containing L-folinic acid (175 mg) (or folinic acid $350 \mathrm{mg}$ ), 5-FU (400 mg/m $\mathrm{m}^{2}$ bolus and $2400 \mathrm{mg} / \mathrm{m}^{2}$ infusion), and oxaliplatin $\left(85 \mathrm{mg} / \mathrm{m}^{2}\right.$ ) (all every 14 days, up to 12 cycles) ${ }^{[47]}$. Only patients with an ECOG performance status of 0 - 1 were eligible for the study, and $72 \%$ of patients in the study cohort had advanced CCA. The use of mFOLFOX led to only a modest prolongation in the median OS, from 5.3 to 6.2 months (HR: 0.69). However, the absolute OS rates at 6 and 12 months were more impressive: at 6 and 12 months, the OS rate for patients in the mFOLFOX 
arm was $50.6 \%$ and $25.9 \%$, respectively, compared with $5.5 \%$ and $11.4 \%$ for active symptom control alone. mFOLFOX was well tolerated, with only a manageable increase in the rate of Grade $\geq 3$ neutropenia and fatigue $^{[47]}$.

The most prominent alternative for mFOLFOX in second-line treatment is a combination of irinotecan and capecitabine (XELIRI). The XELIRI regimen (irinotecan $180 \mathrm{mg} / \mathrm{m}^{2}$ on Day 1 and capecitabine $1000 \mathrm{mg} / \mathrm{m}^{2}$ BID on Days 1-10 of 14-day cycles) was compared to irinotecan alone in a phase II trial that had 60 patients with Gem-Cis pretreated biliary tract cancer ${ }^{[48]}$. In that trial, XELIRI doubled the nine-month OS rate from $32 \%$ to $60.9 \%$ (no significant difference in median OS: 10.1 months vs. 7.3 months; $P=0.107$ ) and increased the disease control rate from $50 \%$ to $63.3 \%$. This benefit came at the cost of only a modest increase in toxicity, but there was a higher rate of palmar-plantar erythrodysesthesia with XELIRI $(6.7 \% v s .0 \%)^{[48]}$.

A third option for advanced CCA patients who failed first-line Gem-Cis is 5-FU-based therapy. Unfortunately, there are no randomized data for comparison of 5-FU to FOLFOX or XELIRI for this indication. The best data come from a large retrospective series of 321 advanced CCA patients ${ }^{[49]}$. In that series, 5-FU-based chemotherapy was modestly effective as second-line chemotherapy for patients with advanced biliary tract cancer who failed on first-line Gem-Cis (ORR 8\% for 5-FU platinum combinations and $1 \%$ for 5-FU alone). A 5-FU-platinum combination was not associated with a better OS or PFS than those outcomes with 5-FU monotherapy ${ }^{[49]}$.

\section{Molecularly targeted therapy for CCA}

CCA has a high level of intra- and intertumoral heterogeneity ${ }^{[50]}$. As a result, clinical trials testing molecularly targeted agents in unselected patients with CCA have consistently yielded negative results. In recent years, however, advances in whole-exome and transcriptome sequencing have shed light on the genetic landscape of the CCA subtypes, opening the door to tailored treatment approaches ${ }^{[51]}$. In fact, recent biomarker-driven clinical trials in CCA patients have reported positive outcomes. These results have prompted FDA approval of pemigatinib as the first targeted treatment for patients with previously treated, advanced CCA who harbor an FGFR2-fusion or rearrangement. We now present an overview of the molecularly targeted agents that are under clinical evaluation in CCA patients.

\section{FGFR-directed therapy}

Gene fusions involving FGFR2 have been reported in $10 \%-20 \%$ of iCCA patients ${ }^{[52,53]}$. The fusions result in constitutive activation of FGFR2, ultimately leading to activation of oncogenesis-promoting signaling pathways, such as RAS-RAF-MEK ${ }^{[54]}$. Several FGFR-targeting agents have been evaluated for the treatment of advanced CCA. As indicated above, pemigatinib recently became the first FDA-approved molecularly targeted agent for treating patients with CCA, specifically those with previously treated tumors and an FGFR2 rearrangement. This approval was based on the results of phase II, multicenter FIGHT-202 study. In that trial, 146 patients with locally advanced or metastatic CCA were treated with pemigatinib at a dose of $13.5 \mathrm{mg} /$ day in a two-weeks on/one-week off schedule ${ }^{[55]}$. Most of the patients $(n=107)$ had an FGFR2 fusion or rearrangement; after a median follow-up of 17.8 months, $36 \%$ of these patients had an objective response to the therapy, and the responses were durable, with a median duration of response of 9.1 months, median PFS of 6.9 months, and median OS an impressive 21.1 months. Grade $\geq 3$ AEs occurred in $64 \%$ of patients in the trial, with $45 \%$ having a serious $\mathrm{AE}$ (most frequently abdominal pain and pyrexia) ${ }^{[55]}$. The clinical benefit of pemigatinib in this setting will be further evaluated in a randomized trial with an active comparator arm.

The pan-FGFR inhibitor infigratinib (BGJ398) was evaluated in a phase II study with CCA patients ${ }^{[56]}$. In that trial, including 61 previously treated patients with advanced CCA and an FGFR alteration, infigratinib induced an ORR of $14.8 \%$ (18.8\% in the cohort of patients with an FGFR2-fusion/alteration). An additional 
$60.6 \%$ of patients experienced disease stabilization under therapy, for an overall disease control rate of 75.4\% (83.3\% in FGFR2-fusion/rearrangement patients). The estimated median PFS in this study was 5.8 months. Grade $\geq 3$ AEs occurred in $41 \%$ of patients, with hyperphosphatemia (16.4\%), stomatitis (6.6\%), and palmar-plantar erythrodysesthesia (4.9\%) being the most common high-grade toxicities ${ }^{[5]}$. A phase III clinical trial comparing infigratinib to Gem-Cis in the first-line treatment of patients with locally advanced/ metastatic CCA and an FGFR2-fusion/rearrangement is ongoing (NCT03773302).

During the 2020 annual meeting of the American Society of Clinical Oncology (ASCO), phase II data were presented for the irreversible FGFR1-4 inhibitor futibatinib ${ }^{[57]}$. In total, 103 patients with unresectable or metastatic iCCA and an FGFR2 fusion (or another rearrangement involving this gene) with disease progression after at least one prior systemic therapy (including Gem-Cis) were treated with futibatinib at a dose of $20 \mathrm{mg} /$ day. The data presented at ASCO included the first 67 patients with at least six months of follow-up. An objective response was obtained in $37.3 \%$ of patients, with an additional $44.8 \%$ of patients experiencing disease stabilization (disease control rate $82.1 \%$ ). Responses also proved to be durable, with a median duration of response of 8.3 months. The median PFS was reported at 7.2 months, with a 6- and 12-month PFS rates of $61.0 \%$ and $39.4 \%$, respectively. Grade $\geq 3$ AEs were reported in $56.7 \%$ of patients, with $10.4 \%$ of treatment-related severe AEs. Only one patient had to discontinue the therapy for reasons of toxicity. The most common Grade 3 AE with futibatinib consisted of hyperphosphatemia $(26.9 \%)^{[57]}$. Based on these excellent results, futibatinib is also being compared to Gem-Cis in a randomized phase III trial, including previously untreated CCA patients (NCT04093362).

In other work, the pan-FGFR inhibitor erdafitinib demonstrated clinical activity in patients with FGFRmutated solid tumors. In a phase I basket trial, erdafitinib induced a partial response in 3 of 11 CCA patients with an FGFR2-fusion or rearrangement ${ }^{[58]}$.

A fifth pan-FGFR inhibitor that demonstrated potential in patients with CCA is derazantinib. In a multicenter phase I/II trial of 29 patients with unresectable iCCA and an FGFR2-fusion, it was associated with an ORR of $20.7 \%$ and a disease control rate of $82.8 \%$. The estimated PFS was 5.7 months, and $27.6 \%$ of patients had Grade $\geq 3 \mathrm{AEs}^{[59]}$. A pivotal trial of derazantinib in patients with iCCA is ongoing (NCT03230318).

\section{IDH-directed therapy}

Mutations in $I D H-1$ and -2 are present in $15 \%-20 \%$ of patients with $\mathrm{iCCA}^{[51,60]}$. These mutations profoundly affect cell differentiation and cell growth, and they are involved in tumorigenesis ${ }^{[61]}$. Several inhibitors of mutant IDH proteins have been developed in recent years. Ivosidenib (AG-120) is a first-in-class, oral, small-molecule inhibitor of the mutant IDH1 protein. In the randomized, phase III ClarIDHy trial, 185 previously treated patients with advanced CCA and an IDH1 mutation were randomly assigned (2:1) to receive either ivosidenib (500 $\mathrm{mg}$ per day) or matching placebo ${ }^{[62]}$. The study met its primary endpoint, with a significantly longer median PFS for patients in the ivosidenib arm than in the placebo arm (2.7 months vs. 1.4 months; $\mathrm{HR}=0.37,95 \% \mathrm{CI}$ : $0.25-0.54, P<0.001$ ). At the 6 - and 12 -month marks, $32 \%$ and $21.9 \%$ of patients, respectively, treated with ivosidenib were free of progression, whereas none of the patients in the placebo arm was progression-free at six months. The ORR with ivosidenib was low (2.4\%), with $50.8 \%$ of patients having disease stabilization. The median OS among patients treated with ivosidenib was 10.8 months. Patients in the placebo arm had a median OS of 9.7 months, but this OS was significantly influenced by $57 \%$ of placebo patients crossing over to ivosidenib. Overall, $46 \%$ of patients experienced a Grade 3/4 AE on ivosidenib as compared with $36 \%$ with placebo; the most common AEs seen with ivosidenib were nausea $(32.1 \%)$, diarrhea $(28.8 \%)$, and fatigue $(23.7 \%)^{[62]}$. The results of phase III ClarIDHy trial are especially important in CCA treatment as they provide level A evidence for the efficacy of targeted therapy in this setting and establish a role for molecular profiling in this cancer type. Several other 
inhibitors of mutant IDH proteins are under clinical evaluation in patients with $I D H$-mutant solid tumors (including CCA), e.g., enasidenib (NCT02273739), IDH305 (NCT02381886), and AG-881 (NCT02481154).

\section{Targeting ROS1 and NTRK fusions}

Gene fusions involving ROS1 have been reported in about $8 \%$ of CCA patients ${ }^{[63]}$, and an oncogenic role for ROS kinase fusions was established in a CCA mouse model ${ }^{[64]}$. These findings make ROS1 an interesting therapeutic target. The ALK and ROS1 inhibitors ceritinib and crizotinib are already being used in patients with metastatic non-small cell lung cancers that harbor ALK and ROS1 fusions. Phase II studies are ongoing to investigate their potential in patients with ROS1- and/or ALK-mutated CCA (NCT02374489 and NCT02034981). Recently, the FDA gave an agnostic approval to the NTRK inhibitor larotrectinib to treat patients with solid tumors harboring an NTRK gene fusion. This approval was based on the results of three multicenter, single-arm trials (LOXO-TRK-1400, SCOUT, and NAVIGATE) of patients with solid tumors and an NTRK fusion. Larotrectinib induced an ORR of $75 \%$, with $71 \%$ of responses ongoing at one year ${ }^{[65]}$. These studies also included two patients with CCA, one of whom experienced disease stabilization under larotrectinib ${ }^{[65]}$. In August 2019, the FDA approved the NTRK inhibitor entrectinib for treating patients with solid tumors and NTRK gene fusions. This approval followed an integrated analysis of the pivotal Phase II STARTRK-2, Phase I STARTRK-1, and Phase I ALKA-372-001 trials ${ }^{[66]}$. In these studies, in which several NTRK-positive advanced CCA were included, entrectinib induced an ORR of 57\%, with a median duration of response of 10 months. Notwithstanding the rarity of NTRK fusions in CCA patients, the fact that this alteration is now actionable with effective targeted therapies justifies screening for it in patients with advanced CCA.

\section{EGFR-directed therapy}

Patients with CCA often harbor mutations in $E G F R^{[52]}$. The mutations are more common in patients with pCCA and dCCA (about 15\%) than in iCCA patients ${ }^{[67]}$. EGFR inhibitors in patients with biliary tract cancer, either as monotherapy or in combination with chemotherapy, have been studied. Unfortunately, the trials consistently yielded disappointing results ${ }^{[68,69]}$. The only phase III trial of EGFR inhibitors in this condition studied the addition of erlotinib to gemcitabine and oxaliplatin as first-line treatment for patients with metastatic biliary cancer; although the response rate was significantly increased, this did not translate into a longer PFS or OS ${ }^{[70]}$. Similarly, the addition of panitumumab to Cis-Gem did not improve the ORR, PFS, or OS in a phase II trial of 62 patients with KRAS-wildtype biliary tract cancer ${ }^{[7]]}$, and no improvement in ORR or PFS was found in a phase II/III TreeTopp trial that evaluated the addition of the pan-HER inhibitor varlitinib to capecitabine as a second-line treatment for patients with biliary tract cancer.

\section{Angiogenesis-directed therapy}

The results of preclinical studies suggest that several angiogenic factors are important in the tumorigenesis of biliary tract cancers ${ }^{[72,73]}$. Thus, angiogenesis-directed therapy has been explored as a therapeutic strategy in patients with these tumor types. In a phase II trial of 35 patients with advanced biliary tract cancer, the combination of GEMOX with bevacizumab had promising antitumor activity (median PFS: seven months; six-month PFS rate: 63\%) with a tolerable safety profile ${ }^{[7]}$. In a randomized phase II trial $(n=57)$, the addition of bevacizumab to GEMOX significantly prolonged the median PFS, from 3.72 to 6.48 months $(P=0.049)$, with only a small increase in toxicity ${ }^{[75]}$. In contrast with those results, a randomized phase II trial, presented during the 2020 annual Gastrointestinal Cancers Symposium, found that the addition of the VEGF inhibitor ramucirumab did not improve the ORR, PFS, or OS in patients with biliary tract cancer ${ }^{[76]}$. Similarly, a phase I study evaluating a combination of ramucirumab and the immune checkpoint inhibitor pembrolizumab found a limited clinical effect in patients with previously treated biliary tract cancer ${ }^{[77]}$.

\section{Emerging targets}

Several other promising new drugs are in early clinical development for the treatment of patients with CCA. Constituted JAK/STAT activation is a recurrent finding in CCA, making it a potential therapeutic 
target ${ }^{[78,79]}$. In a phase I trial, the STAT3 inhibitor ABC294640 showed activity in CCA, and the inhibitor is under further evaluation in a phase II trial (NCT03377179).

Amplification and overexpression of MET have been described in CCA, with associated poor prognosis ${ }^{[80]}$. Clinical studies evaluating MET inhibitors in monotherapy revealed limited clinical activity. In contrast, phase I data of a study evaluating the combination of the MET inhibitor tivantinib with gemcitabine in patients with metastatic solid cancers suggest the presence of antitumor activity ${ }^{[8]]}$. However, phase II data failed to show an ORR, PFS, or OS benefit from the addition of the MET inhibitor merestinib to first-line Gem-Cis in patients with advanced biliary tract cancer ${ }^{[76]}$.

\section{IMMUNE CHECKPOINT INHIBITION FOR CCA}

Over the last decade, immune checkpoint inhibitors have revolutionized the treatment landscape of many different cancer types, and this strategy is being explored in CCA.

Pembrolizumab is a monoclonal antibody directed against the programmed death-1 receptor (PD-1). In 2017, the FDA had approved pembrolizumab for the treatment of microsatellite instability-high solid tumors. In the phase II Keynote-158 trial, the antibody had robust clinical activity in patients with noncolorectal microsatellite instability-high/mismatch repair-deficient solid tumors. Two hundred and thirty-three patients, including 22 with advanced CCA, were enrolled in the study. Pembrolizumab was administered at a dose of $200 \mathrm{mg}$ once every three weeks for a maximum of two years ${ }^{[82]}$. An overall response rate of $34.3 \%$ was reported, with a median duration of response that was not yet reached after a median follow-up of 13.4 months. Among the cohort of CCA patients, the ORR was 49\%, and two patients had a complete response; the median OS in CCA patients was 24.3 months. Treatment-related AEs most commonly fatigue (14.6\%), pruritus (12.9\%), and diarrhea (12.0\%) - occurred in $64.8 \%$ of patients; Grade $\geq 3$ treatment-related AEs occurred in $14.6 \%$ of patients, and $23.2 \%$ experienced an immune-related $\mathrm{AE}^{[82]}$. Thus, these data establish pembrolizumab as an effective and safe treatment option for patients with CCA and mismatch repair deficiency. According to Silva et al. ${ }^{[83]}, 5 \%-10 \%$ of patients with CCA meet this criterion.

Pembrolizumab was also evaluated in non-mismatch repair-deficient CCA patients. In the large multicohort, phase Ib Keynote-028 trial, 24 patients with PD-L1-positive CCA were treated with pembrolizumab at a dose of $10 \mathrm{mg} / \mathrm{kg}$ every two weeks for up to two years ${ }^{[84]}$. In this cohort, four patients (17\%), three with CCA and one with gallbladder cancer, had a partial response, and four patients (17\%) had disease stabilization; at 12 months, $27.6 \%$ of patients were still alive. The rate of Grade 3 toxicities was $16.7 \%$, with no reported Grade $\geq 4$ toxicities ${ }^{[84]}$.

The PD-1 inhibitor nivolumab has also been evaluated in patients with biliary tract cancer. In a study of 30 patients with metastatic disease, nivolumab was associated with an ORR of $20 \%$ and a disease control rate of $60 \%$; the median PFS was 3.1 months ${ }^{[85]}$. Preliminary results of an ongoing phase II trial in patients with advanced refractory biliary tract cancer indicate an ORR of $22 \%$, with a disease control rate of $60 \%$. The median OS in this study was 14.2 months, with 6- and 12 -month OS rates of $71.4 \%$ and $52.3 \%$, respectively. At six months, $35.2 \%$ of patients were free of progression; the rate was $24.1 \%$ at 12 months. The safety profile was in line with that of previous reports on nivolumab. Importantly, this trial did not select for PDL1 expression at study entry ${ }^{[86]}$. In a Japanese trial, nivolumab was evaluated as monotherapy or combined with chemotherapy in 60 patients with biliary tract cancer; the monotherapy was associated with a median OS of 5.2 months, a median PFS of 1.4 months, and a low ORR, with only one patient obtaining a response. In the combined therapy cohort, median OS (5.4 months) and median PFS (4.2 months) were longer, and 11 of 30 patients had an objective response ${ }^{[87]}$. 
A third immune checkpoint inhibitor under evaluation in CCA is the PD-L1 inhibitor durvalumab. In a phase I trial, durvalumab was evaluated as monotherapy $(n=42)$ or in combination with the CTLA4 inhibitor tremelimumab $(n=65)$ to treat patients with previously advanced biliary tract cancer ${ }^{[88]}$. At 12 weeks, durvalumab monotherapy was associated with a disease control rate of $16.7 \%$; with the durvalumab-tremelimumab combination, this metric increased to $32.2 \%$. The median duration of response with durvalumab alone was 9.7 months; with the combination, it was 8.5 months. The median OS of patients in the monotherapy cohort was 8.1 months; with durvalumab plus tremelimumab, it was 10.1 months. The treatments were generally well-tolerated, with Grade $\geq 3$ treatment-related AEs in $19 \%$ and $23 \%$ of patients treated with monotherapy and combination, respectively ${ }^{[88]}$. Thus, these findings reveal promising clinical activity of durvalumab, both as monotherapy and in combination with tremelimumab in patients with advanced biliary tract cancer. Durvalumab is also being studied in combination with chemotherapy: in the randomized phase III TOPAZ trial, the combination of durvalumab with Gem-Cis is under evaluation as a first-line treatment for patients with advanced biliary cancer (NCT03875235).

\section{CONCLUSION}

Patients with advanced CCA face a poor prognosis. The standard of care for these patients is gemcitabinebased doublet chemotherapy (Gen-Cis or GemS), which has a median OS of about one year. For patients with disease progression after first-line therapy, there is no universal standard of care. Small steps have been made towards a personalized treatment approach for patients with CCA. The most promising approach is the recently FDA-approved FGFR inhibitor pemigatinib in the second-line treatment of patients with previously treated advanced CCA harboring an FGFR2 fusion or rearrangement. For patients with an IDH1 mutation, ivosidenib treatment has been found to show progression-free efficacy. Several other targeted therapies are being explored in molecularly oriented clinical trials of CCA: promising data have been generated with the immune checkpoint inhibitors pembrolizumab, nivolumab, and durvalumab in patients with advanced CCA, and it appears that immunotherapy will become an important strategy in the treatment of these patients. The response of mismatch repair-deficient CCA patients to pembrolizumab treatment is especially promising.

\section{DECLARATIONS}

\section{Authors' contributions}

Made equal and substantial contribution to the conception of idea, literature review, and drafting and finalization of manuscript: Alqahtani SA, Colombo M

\section{Availability of data and materials}

Not applicable.

\section{Financial support and sponsorship}

None.

\section{Conflicts of interest}

Both authors declared that there are no conflicts of interest.

\section{Ethical approval and consent to participate}

Not applicable.

\section{Consent for publication}

Not applicable. 


\section{Copyright}

(c) The Author(s) 2020.

\section{REFERENCES}

1. Razumilava N, Gores GJ. Cholangiocarcinoma. Lancet 2014;383:2168-79.

2. DeOliveira ML, Cunningham SC, Cameron JL, Kamangar F, Winter JM, et al. Cholangiocarcinoma: thirty-one-year experience with 564 patients at a single institution. Ann Surg 2007;245:755-62.

3. Valle JW, Borbath I, Khan SA, Huguet F, Gruenberger T, et al. Biliary cancer: ESMO Clinical Practice Guidelines for diagnosis, treatment and follow-up. Ann Oncol 2016;27:v28-37.

4. Nakanuma Y, Sato Y, Harada K, Sasaki M, Xu J, et al. Pathological classification of intrahepatic cholangiocarcinoma based on a new concept. World J Hepatol 2010;2:419-27.

5. Petrick JL, Yang B, Altekruse SF, Van Dyke AL, Koshiol J, et al. Risk factors for intrahepatic and extrahepatic cholangiocarcinoma in the United States: a population-based study in SEER-Medicare. PLoS One 2017;12:e0186643.

6. Massarweh NN, El-Serag HB. Epidemiology of hepatocellular carcinoma and intrahepatic cholangiocarcinoma. Cancer Control 2017;24:1073274817729245.

7. Palmer WC, Patel T. Are common factors involved in the pathogenesis of primary liver cancers? A meta-analysis of risk factors for intrahepatic cholangiocarcinoma. J Hepatol 2012;57:69-76.

8. Lee BS, Cha BH, Park EC, Roh J. Risk factors for perihilar cholangiocarcinoma: a hospital-based case-control study. Liver Int 2015;35:1048-53.

9. Massironi S, Pilla L, Elvevi A, Longarini R, Rossi RE, et al. New and emerging systemic therapeutic options for advanced cholangiocarcinoma. Cells 2020;9:688.

10. Tyson GL, El-Serag HB. Risk factors for cholangiocarcinoma. Hepatology 2011;54:173-84.

11. Endo I, Gonen M, Yopp AC, Dalal KM, Zhou Q, et al. Intrahepatic cholangiocarcinoma: rising frequency, improved survival, and determinants of outcome after resection. Ann Surg 2008;248:84-96.

12. Edeline J, Benabdelghani M, Bertaut A, Watelet J, Hammel P, et al. Gemcitabine and oxaliplatin chemotherapy or surveillance in resected biliary tract cancer (PRODIGE 12-ACCORD 18-UNICANCER GI): a randomized phase III study. J Clin Oncol 2019;37:658-67.

13. Ebata T, Hirano S, Konishi M, Uesaka K, Tsuchiya Y, et al. Randomized clinical trial of adjuvant gemcitabine chemotherapy versus observation in resected bile duct cancer. Br J Surg 2018;105:192-202.

14. Primrose JN, Fox RP, Palmer DH, Malik HZ, Prasad R, et al. Capecitabine compared with observation in resected biliary tract cancer (BILCAP): a randomised, controlled, multicentre, phase 3 study. Lancet Oncol 2019;20:663-73.

15. Stein A, Arnold D, Bridgewater J, Goldstein D, Jensen LH, et al. Adjuvant chemotherapy with gemcitabine and cisplatin compared to observation after curative intent resection of cholangiocarcinoma and muscle invasive gallbladder carcinoma (ACTICCA-1 trial) - a randomized, multidisciplinary, multinational phase III trial. BMC Cancer 2015;15:564.

16. Horgan AM, Amir E, Walter T, Knox JJ. Adjuvant therapy in the treatment of biliary tract cancer: a systematic review and meta-analysis. J Clin Oncol 2012;30:1934-40.

17. Kiefer MV, Albert M, McNally M, Robertson M, Sun W, et al. Chemoembolization of intrahepatic cholangiocarcinoma with cisplatinum, doxorubicin, mitomycin C, ethiodol, and polyvinyl alcohol: a 2-center study. Cancer 2011;117:1498-505.

18. Kuhlmann JB, Euringer W, Spangenberg HC, Breidert M, Blum HE, et al. Treatment of unresectable cholangiocarcinoma: conventional transarterial chemoembolization compared with drug eluting bead-transarterial chemoembolization and systemic chemotherapy. Eur J Gastroenterol Hepatol 2012;24:437-43.

19. Park SY, Kim JH, Yoon HJ, Lee IS, Yoon HK, et al. Transarterial chemoembolization versus supportive therapy in the palliative treatment of unresectable intrahepatic cholangiocarcinoma. Clin Radiol 2011;66:322-8.

20. Hoffmann RT, Paprottka PM, Schön A, Bamberg F, Haug A, et al. Transarterial hepatic yttrium-90 radioembolization in patients with unresectable intrahepatic cholangiocarcinoma: factors associated with prolonged survival. Cardiovasc Intervent Radiol 2012;35:105-16.

21. Rafi S, Piduru SM, El-Rayes B, Kauh JS, Kooby DA, et al. Yttrium-90 radioembolization for unresectable standard-chemorefractory intrahepatic cholangiocarcinoma: survival, efficacy, and safety study. Cardiovasc Intervent Radiol 2013;36:440-8.

22. Ghidini M, Tomasello G, Botticelli A, Barni S, Zabbialini G, et al. Adjuvant chemotherapy for resected biliary tract cancers: a systematic review and meta-analysis. HPB (Oxford) 2017;19:741-8.

23. Shroff RT, Kennedy EB, Bachini M, Bekaii-Saab T, Crane C, et al. Adjuvant therapy for resected biliary tract cancer: ASCO clinical practice guideline. J Clin Oncol 2019;37:1015-27.

24. Glimelius B, Hoffman K, Sjödén PO, Jacobsson G, Sellström H, et al. Chemotherapy improves survival and quality of life in advanced pancreatic and biliary cancer. Ann Oncol 1996;7:593-600.

25. Park JS, Oh SY, Kim SH, Kwon HC, Kim JS, et al. Single-agent gemcitabine in the treatment of advanced biliary tract cancers: a phase II study. Jpn J Clin Oncol 2005;35:68-73.

26. Suzuki E, Furuse J, Ikeda M, Okusaka T, Nakachi K, et al. Treatment efficacy/safety and prognostic factors in patients with advanced biliary tract cancer receiving gemcitabine monotherapy: an analysis of 100 cases. Oncology 2010;79:39-45.

27. Thongprasert S, Napapan S, Charoentum C, Moonprakan S. Phase II study of gemcitabine and cisplatin as first-line chemotherapy in inoperable biliary tract carcinoma. Ann Oncol 2005;16:279-81.

28. Giuliani F, Gebbia V, Maiello E, Borsellino N, Bajardi E, et al. Gemcitabine and cisplatin for inoperable and/or metastatic biliary tree 
carcinomas: a multicenter phase II study of the Gruppo Oncologico dell'Italia Meridionale (GOIM). Ann Oncol 2006;17:vii73-7.

29. Valle J, Wasan H, Palmer DH, Cunningham D, Anthoney A, et al. Cisplatin plus gemcitabine versus gemcitabine for biliary tract cancer. N Engl J Med 2010;362:1273-81.

30. Okusaka T, Nakachi K, Fukutomi A, Mizuno N, Ohkawa S, et al. Gemcitabine alone or in combination with cisplatin in patients with biliary tract cancer: a comparative multicentre study in Japan. Br J Cancer 2010;103:469-74.

31. Valle JW, Furuse J, Jitlal M, Beare S, Mizuno N, et al. Cisplatin and gemcitabine for advanced biliary tract cancer: a meta-analysis of two randomised trials. Ann Oncol 2014;25:391-8.

32. Morizane C, Okusaka T, Mizusawa J, Katayama H, Ueno M, et al. Combination gemcitabine plus S-1 versus gemcitabine plus cisplatin for advanced/recurrent biliary tract cancer: the FUGA-BT (JCOG1113) randomized phase III clinical trial. Ann Oncol 2019;30:1950-8.

33. André T, Tournigand C, Rosmorduc O, Provent S, Maindrault-Goebel F, et al. Gemcitabine combined with oxaliplatin (GEMOX) in advanced biliary tract adenocarcinoma: a GERCOR study. Ann Oncol 2004;15:1339-43.

34. Kim ST, Kang JH, Lee J, Lee HW, Oh SY, et al. Capecitabine plus oxaliplatin versus gemcitabine plus oxaliplatin as first-line therapy for advanced biliary tract cancers: a multicenter, open-label, randomized, phase III, noninferiority trial. Ann Oncol 2019;30:788-95.

35. Sahai V, Catalano PJ, Zalupski MM, Lubner SJ, Menge MR, et al. Nab-paclitaxel and gemcitabine as first-line treatment of advanced or metastatic cholangiocarcinoma: a phase 2 clinical trial. JAMA Oncol 2018;4:1707-12.

36. Shroff RT, Javle MM, Xiao L, Kaseb AO, Varadhachary GR, et al. Gemcitabine, cisplatin, and nab-paclitaxel for the treatment of advanced biliary tract cancers: a phase 2 clinical trial. JAMA Oncol 2019;5:824-30.

37. Sakai D, Kanai M, Kobayashi S, Eguchi H, Baba H, et al. 615ORandomized phase III study of gemcitabine, cisplatin plus S-1 (GCS) versus gemcitabine, cisplatin (GC) for advanced biliary tract cancer (KHBO1401-MITSUBA). Ann Oncol 2018;29.

38. Takada T, Kato H, Matsushiro T, Nimura Y, Nagakawa T, et al. Comparison of 5-fluorouracil, doxorubicin and mitomycin C with 5-fluorouracil alone in the treatment of pancreatic-biliary carcinomas. Oncology 1994;51:396-400.

39. Choi CW, Choi IK, Seo JH, Kim BS, Kim JS, et al. Effects of 5-fluorouracil and leucovorin in the treatment of pancreatic-biliary tract adenocarcinomas. Am J Clin Oncol 2000;23:425-8.

40. Chen JS, Jan YY, Lin YC, Wang HM, Chang WC, et al. Weekly $24 \mathrm{~h}$ infusion of high-dose 5-fluorouracil and leucovorin in patients with biliary tract carcinomas. Anticancer Drugs 1998;9:393-7.

41. Petekkaya I, Gezgen G, Roach EC, Solak M, Gullu I. Long-term advanced cholangiocarcinoma survivor with single-agent capecitabine. J buon 2012;17:796.

42. Schweitzer N, Kirstein MM, Kratzel AM, Mederacke YS, Fischer M, et al. Second-line chemotherapy in biliary tract cancer: Outcome and prognostic factors. Liver Int 2019;39:914-23.

43. Fornaro L, Cereda S, Aprile G, Di Girolamo S, Santini D, et al. Multivariate prognostic factors analysis for second-line chemotherapy in advanced biliary tract cancer. Br J Cancer 2014;110:2165-9.

44. Brieau B, Dahan L, De Rycke Y, Boussaha T, Vasseur P, et al. Second-line chemotherapy for advanced biliary tract cancer after failure of the gemcitabine-platinum combination: a large multicenter study by the Association des Gastro-Entérologues Oncologues. Cancer 2015;121:3290-7.

45. Neuzillet C, Casadei Gardini A, Brieau B, Vivaldi C, Smolenschi C, et al. Prediction of survival with second-line therapy in biliary tract cancer: actualisation of the AGEO CT2BIL cohort and European multicentre validations. Eur J Cancer 2019;111:94-106.

46. Lamarca A, Hubner RA, David Ryder W, Valle JW. Second-line chemotherapy in advanced biliary cancer: a systematic review. Ann Oncol 2014;25:2328-38.

47. Lamarca A, Palmer DH, Wasan HS, Ross PJ, Ma YT, et al. ABC-06 | A randomised phase III, multi-centre, open-label study of active symptom control (ASC) alone or ASC with oxaliplatin / 5-FU chemotherapy (ASC+mFOLFOX) for patients (pts) with locally advanced / metastatic biliary tract cancers (ABC) previously-treated with cisplatin/gemcitabine (CisGem) chemotherapy. J Clin Oncol 2019;37:4003.

48. Zheng Y, Tu X, Zhao P, Jiang W, Liu L, et al. A randomised phase II study of second-line XELIRI regimen versus irinotecan monotherapy in advanced biliary tract cancer patients progressed on gemcitabine and cisplatin. Br J Cancer 2018;119:291-5.

49. Kim BJ, Yoo C, Kim KP, Hyung J, Park SJ, et al. Efficacy of fluoropyrimidine-based chemotherapy in patients with advanced biliary tract cancer after failure of gemcitabine plus cisplatin: retrospective analysis of 321 patients. Br J Cancer 2017;116:561-7.

50. Nakamura H, Arai Y, Totoki Y, Shirota T, Elzawahry A, et al. Genomic spectra of biliary tract cancer. Nat Genet 2015;47:1003-10.

51. Rizvi S, Khan SA, Hallemeier CL, Kelley RK, Gores GJ. Cholangiocarcinoma - evolving concepts and therapeutic strategies. Nat Rev Clin Oncol 2018;15:95-111.

52. Churi CR, Shroff R, Wang Y, Rashid A, Kang HC, et al. Mutation profiling in cholangiocarcinoma: prognostic and therapeutic implications. PLoS One 2014;9:e115383.

53. Ross JS, Wang K, Gay L, Al-Rohil R, Rand JV, et al. New routes to targeted therapy of intrahepatic cholangiocarcinomas revealed by next-generation sequencing. Oncologist 2014;19:235-42.

54. Wu YM, Su F, Kalyana-Sundaram S, Khazanov N, Ateeq B, et al. Identification of targetable FGFR gene fusions in diverse cancers. Cancer Discov 2013;3:636-47.

55. Abou-Alfa GK, Sahai V, Hollebecque A, Vaccaro G, Melisi D, et al. Pemigatinib for previously treated, locally advanced or metastatic cholangiocarcinoma: a multicentre, open-label, phase 2 study. Lancet Oncol 2020;21:671-84.

56. Javle M, Lowery M, Shroff RT, Weiss KH, Springfeld C, et al. Phase II study of BGJ398 in patients with FGFR-altered advanced cholangiocarcinoma. J Clin Oncol 2018;36:276-82.

57. Goyal L, Meric-Bernstein F, Hollebecque A, Valle J, Morizane C, et al. FOENIX-CCA2: a phase II, open-label, multicenter study of futibatinib in patients (pts) with intrahepatic cholangiocarcinoma (iCCA) harboring FGFR2 gene fusions or other rearrangements. J Clin 
Oncol 2020;38:108.

58. Nishina T, Takahashi S, Iwasawa R, Noguchi H, Aoki M, et al. Safety, pharmacokinetic, and pharmacodynamics of erdafitinib, a panfibroblast growth factor receptor (FGFR) tyrosine kinase inhibitor, in patients with advanced or refractory solid tumors. Invest New Drugs 2018;36:424-34.

59. Mazzaferro V, El-Rayes BF, Droz Dit Busset M, Cotsoglou C, Harris WP, et al. Derazantinib (ARQ 087) in advanced or inoperable FGFR2 gene fusion-positive intrahepatic cholangiocarcinoma. Br J Cancer 2019;120:165-71.

60. Borger DR, Tanabe KK, Fan KC, Lopez HU, Fantin VR, et al. Frequent mutation of isocitrate dehydrogenase (IDH)1 and IDH2 in cholangiocarcinoma identified through broad-based tumor genotyping. Oncologist 2012;17:72-9.

61. Dang L, Yen K, Attar EC. IDH mutations in cancer and progress toward development of targeted therapeutics. Ann Oncol 2016;27:599608.

62. Abou-Alfa GK, Macarulla T, Javle M, Kelley RK, Lubner SJ, et al. ClarIDHy: a global, phase 3, randomized, double-blind study of ivosidenib (IVO) vs placebo in patients with advanced cholangiocarcinoma (CC) with an isocitrate dehydrogenase 1 (IDH1) mutation. Ann Oncol 2019;30:Abstract LBA 10_PR.

63. Gu TL, Deng X, Huang F, Tucker M, Crosby K, et al. Survey of tyrosine kinase signaling reveals ROS kinase fusions in human cholangiocarcinoma. PLoS One 2011;6:e15640.

64. Saborowski A, Saborowski M, Davare MA, Druker BJ, Klimstra DS, et al. Mouse model of intrahepatic cholangiocarcinoma validates FIG-ROS as a potent fusion oncogene and therapeutic target. Proc Natl Acad Sci U S A 2013;110:19513-8.

65. Drilon A, Laetsch TW, Kummar S, DuBois SG, Lassen UN, et al. Efficacy of larotrectinib in TRK fusion-positive cancers in adults and children. N Engl J Med 2018;378:731-9.

66. Doebele RC, Drilon A, Paz-Ares L, Siena S, Shaw AT, et al. Entrectinib in patients with advanced or metastatic NTRK fusion-positive solid tumours: integrated analysis of three phase 1-2 trials. Lancet Oncol 2020;21:271-82.

67. Galdy S, Lamarca A, McNamara MG, Hubner RA, Cella CA, et al. HER2/HER3 pathway in biliary tract malignancies; systematic review and meta-analysis: a potential therapeutic target? Cancer Metastasis Rev 2017;36:141-57.

68. Jensen LH. Clinical aspects and perspectives of erlotinib in the treatment of patients with biliary tract cancer. Expert Opin Investig Drugs 2016;25:359-65.

69. Rizzo A, Frega G, Ricci AD, Palloni A, Abbati F, et al. Anti-EGFR monoclonal antibodies in advanced biliary tract cancer: a systematic review and meta-analysis. In Vivo 2020;34:479-88.

70. Lee J, Park SH, Chang HM, Kim JS, Choi HJ, et al. Gemcitabine and oxaliplatin with or without erlotinib in advanced biliary-tract cancer: a multicentre, open-label, randomised, phase 3 study. Lancet Oncol 2012;13:181-8.

71. Vogel A, Kasper S, Bitzer M, Block A, Sinn M, et al. PICCA study: panitumumab in combination with cisplatin/gemcitabine chemotherapy in KRAS wild-type patients with biliary cancer-a randomised biomarker-driven clinical phase II AIO study. Eur J Cancer 2018;92:11-9.

72. Möbius C, Demuth C, Aigner T, Wiedmann M, Wittekind C, et al. Evaluation of VEGF A expression and microvascular density as prognostic factors in extrahepatic cholangiocarcinoma. Eur J Surg Oncol 2007;33:1025-9.

73. Benckert C, Jonas S, Cramer T, Von Marschall Z, Schäfer G, et al. Transforming growth factor beta 1 stimulates vascular endothelial growth factor gene transcription in human cholangiocellular carcinoma cells. Cancer Res 2003;63:1083-92.

74. Zhu AX, Meyerhardt JA, Blaszkowsky LS, Kambadakone AR, Muzikansky A, et al. Efficacy and safety of gemcitabine, oxaliplatin, and bevacizumab in advanced biliary-tract cancers and correlation of changes in 18-fluorodeoxyglucose PET with clinical outcome: a phase 2 study. Lancet Oncol 2010;11:48-54.

75. Bréchon M, Dior M, Dréanic J, Brieau B, Guillaumot MA, et al. Addition of an antiangiogenic therapy, bevacizumab, to gemcitabine plus oxaliplatin improves survival in advanced biliary tract cancers. Invest New Drugs 2018;36:156-62.

76. Valle J, Bai LY, Orlova R, Van Cutsem E, Adeva J, et al. Ramucirumab (RAM) or merestinib (MER) or placebo (PL) plus gemcitabine (GEM) and cisplatin (CIS) as first-line treatment for advanced or metastatic biliary tract cancer (BTC): a randomized, double-blind, phase II study. J Clin Oncol 2020;38:Abstract 477.

77. Arkenau HT, Martin-Liberal J, Calvo E, Penel N, Krebs MG, et al. Ramucirumab plus pembrolizumab in patients with previously treated advanced or metastatic biliary tract cancer: nonrandomized, open-label, phase I trial (JVDF). Oncologist 2018;23:1407-e136.

78. Yang XW, Li L, Hou GJ, Yan XZ, Xu QG, et al. STAT3 overexpression promotes metastasis in intrahepatic cholangiocarcinoma and correlates negatively with surgical outcome. Oncotarget 2017;8:7710-21.

79. Dokduang H, Techasen A, Namwat N, Khuntikeo N, Pairojkul C, et al. STATs profiling reveals predominantly-activated STAT3 in cholangiocarcinoma genesis and progression. J Hepatobiliary Pancreat Sci 2014;21:767-76.

80. Miyamoto M, Ojima H, Iwasaki M, Shimizu H, Kokubu A, et al. Prognostic significance of overexpression of c-Met oncoprotein in cholangiocarcinoma. Br J Cancer 2011;105:131-8.

81. Pant S, Saleh M, Bendell J, Infante JR, Jones S, et al. A phase I dose escalation study of oral c-MET inhibitor tivantinib (ARQ 197) in combination with gemcitabine in patients with solid tumors. Ann Oncol 2014;25:1416-21.

82. Marabelle A, Le DT, Ascierto PA, Di Giacomo AM, De Jesus-Acosta A, et al. Efficacy of pembrolizumab in patients with noncolorectal high microsatellite instability/mismatch repair-deficient cancer: results from the phase II KEYNOTE-158 study. J Clin Oncol 2020;38:110.

83. Silva VW, Askan G, Daniel TD, Lowery M, Klimstra DS, et al. Biliary carcinomas: pathology and the role of DNA mismatch repair deficiency. Chin Clin Oncol 2016;5:62.

84. Bang YJ, Ueno M, Malka D, Chung HC, Nagrial A, et al. Pembrolizumab (pembro) for advanced biliary adenocarcinoma: results from 
the KEYNOTE-028 (KN028) and KEYNOTE-158 (KN158) basket studies. J Clin Oncol 2019;37:4079.

85. Gou M, Zhang Y, Si H, Dai G. Efficacy and safety of nivolumab for metastatic biliary tract cancer. Onco Targets Ther 2019;12:861-7.

86. Kim RD, Kim DW, Alese OB, Li D, Shah N, et al. A phase II study of nivolumab in patients with advanced refractory biliary tract cancers (BTC). J Clin Oncol 2019;37:4097.

87. Ueno M, Ikeda M, Morizane C, Kobayashi S, Ohno I, et al. Nivolumab alone or in combination with cisplatin plus gemcitabine in Japanese patients with unresectable or recurrent biliary tract cancer: a non-randomised, multicentre, open-label, phase 1 study. Lancet Gastroenterol Hepatol 2019;4:611-21.

88. Ioka T, Ueno M, Oh DY, Fujiwara Y, Chen JS, et al. Evaluation of safety and tolerability of durvalumab (D) with or without tremelimumab (T) in patients (pts) with biliary tract cancer (BTC). J Clin Oncol 2019;37:387. 